\title{
Does executive impairment define a frontal variant of Alzheimer's disease?
}

\author{
Michael Woodward, ${ }^{1}$ Henry Brodaty, ${ }^{2,3}$ Karyn Boundy, ${ }^{4}$ David Ames, ${ }^{5}$ Greg Blanch ${ }^{6}$ \\ and Robert Balshaw ${ }^{7}$ on behalf of PRIME Study Group \\ ${ }^{1}$ Aged Care Services, Heidelberg Repatriation Hospital, Austin Health, Heidelberg, Victoria, Australia \\ ${ }^{2}$ Prince of Wales Hospital, Randwick, Sydney, New South Wales, Australia \\ ${ }^{3}$ Dementia Collaborative Research Centre, School of Psychiatry, University of New South Wales, Sydney, New South Wales, Australia \\ ${ }^{4}$ The Queen Elizabeth Hospital, Woodville South, South Australia, Australia \\ ${ }^{5}$ National Ageing Research Institute, Melbourne, Victoria, Australia \\ ${ }^{6}$ Medical Communications, Fanssen-Cilag Pty Ltd, Sydney, New South Wales, Australia \\ ${ }^{7}$ Syreon Corporation, Vancouver, Canada
}

Background: People with Alzheimer's disease (AD) who present with prominent frontal features such as a dysexecutive syndrome may be difficult to differentiate clinically from subjects with frontotemporal lobar degeneration (FTLD). This study was performed to improve the differential diagnosis between $\mathrm{AD}$ and FTLD and to better characterize the AD subgroup with greater executive dysfunction.

Methods: Using a well-defined prospectively studied cohort of cognitively impaired subjects, which included those with $\mathrm{AD}$ and with FTLD, we nominated a frontal variant of $\mathrm{AD}$ (FvAD) group as those $\mathrm{AD}$ subjects with the lowest quartile of scores on the Frontal Assessment Battery (FAB), indicating greatest executive dysfunction, and compared them with the rest of the $\mathrm{AD}$ cases (whom we called the $\mathrm{AD}$ group) and those with FTLD across several baseline variables including cognitive, functional and behavioral scales. We also compared the changes from baseline for these three groups at 6 and 12 months. Additionally, we controlled for dementia severity by matching AD and FTLD cases on a functional scale, the SMAF, and repeated the same comparisons with these severity-matched groups.

Results: The 114 FvAD subjects had a mean age of 78.1 years and Mini-mental State Examination (MMSE) scores of 16.6, and the (remaining) AD group had a mean age of 78.4 years and MMSE of 22.4. There were 30 FTLD subjects with a mean age at baseline of 70.9 years and a mean baseline MMSE of 23.4. The FvAD group was significantly more severely impaired than the other two groups on all baseline assessments except the behavioral scale, the Neuropsychiatric Inventory (NPI), where there was insignificantly less impairment than in the FTLD group. In the analysis of subjects matched at baseline for functional impairment, the FvAD and FTLD groups were not significantly different on most assessment scales although on the FAB, clockdrawing and MMSE the FvAD subjects were still significantly more impaired. These two severity-matched groups were also similar in other baseline characteristics except for older age and less psychotropic use in the FvAD group. The severity-matched FvAD group was significantly different from the AD group in almost all assessment scales. All three unmatched and matched groups declined similarly over 12 months.

Conclusions: When groups were not matched for baseline severity, the use of the FAB defined a group of $\mathrm{AD}$ subjects with greater executive dysfunction that were distinguished from both the remainder of the $\mathrm{AD}$ and FTLD subjects in almost all domains except behavioral disturbance and probably were just more severely affected $\mathrm{AD}$ subjects. The $\mathrm{FAB}$ is thus more useful as a marker of dementia severity than as a scale to detect a frontal variant of $\mathrm{AD}$ or to distinguish $\mathrm{AD}$ from FTLD. Controlling for severity, however, did allow the definition of a subgroup of AD subjects that more closely resembled FTLD subjects than the remainder of the $\mathrm{AD}$ subjects. It is proposed that subjects with dementia presenting with greater executive impairment but without prominent behavioral symptoms are likely to have AD rather than FTLD, especially if they are quite functionally impaired. With time FTLD subjects develop increasing executive dysfunction and increasingly resemble the more severely affected $\mathrm{AD}$ subjects.

Key words: dementia, Alzheimer's disease, frontotemporal dementia, executive dysfunction, behavioral disturbance, multicenter database

Correspondence should be addressed to: Michael Woodward, Austin Health/University of Melbourne, Heidelberg Repatriation Hospital, Banksia Street, Heidelberg West, VIC 3081, Australia. Phone: +61 394962185; Fax: +61 394962613. Email: Michael.woodward@austin.org.au. Received 7 Dec
2009; revision requested 8 Feb 2010; revised version received 18 Apr 2010; further revision requested 15 Jun 2010; further revised version received 28 Jun 2010; accepted 29 Jun 2010. First published online 19 August 2010. 


\section{Introduction}

Correctly diagnosing dementia type is increasingly important in an era when potentially diseasemodifying agents are soon likely to be marketed. Alzheimer's disease (AD) affects a range of cognitive domains including memory, executive functions, language and visuospatial function. Affected individuals show a range of impairment profiles across these functions, and these impairments may progress differently in each domain. Characteristically memory is affected early in AD (Grady et al., 1988; Welsh et al., 1991; Greene et al., 1996) and in severe AD all domains are affected (Price et al., 1993). Atypical presentations of $\mathrm{AD}$ are, however, recognized and relate closely to the pattern of pathology at autopsy (Kanne et al., 1998; Galton et al., 2000). Impairments of executive and language functions may be early prominent features of $\mathrm{AD}$ (Becker et al., 1988; Binetti et al., 1996; Gorno-Tempini et al., 2008) while behavioral abnormalities may also manifest early and have prognostic value (Stern et al., 1987; Mega et al., 1996). A frontal variant of $\mathrm{AD}$ with a neuropathological correlate of greater numbers of neurofibrillary tangles within the frontal lobes than is seen in other AD patients is reported (Johnson et al., 1999).

It can be difficult to distinguish $\mathrm{AD}$ patients with disproportionate frontal features from patients with frontotemporal dementia (FTD) - now known as frontotemporal lobar degeneration (FTLD) (Lebert et al., 1998; Storey et al., 2002). The NINCDS-ADRDA diagnostic criteria for AD have low specificity when comparing AD with FTLD patients, where most FTLD patients can fulfill NINCDS-ADRDA criteria for AD (Varma et al., 1999). Additionally, the Lund-Manchester clinical criteria for FTLD frequently misdiagnose $\mathrm{AD}$, with $34 \%$ of clinically diagnosed $\mathrm{AD}$ cases in a community sample of 185 dementia cases fulfilling these non-AD criteria (Ikeda et al., 2004). This clinical difficulty in diagnosis may reflect the underlying pathology, with $7-32 \%$ of patients clinically diagnosed as having FTLD found to have $\mathrm{AD}$ pathology alone or in combination with other pathology at autopsy (Kertesz et al., 2005; Knopmann et al., 2005; Forman et al., 2005; Knibb et al., 2006). An autopsy study of 45 well-characterized subjects with dementia had eight cases with both AD and FTLD pathology (Woodward et al., 2010a) and more recently the Alzheimer's Disease Neuroimaging Initiative reported that one of the first nine cases to come to autopsy had coexistent AD and FTLD (TDP-43) pathology (Cairns et al., 2010). Patients with FTLD may have prominent early memory loss, further blurring the distinction between FTLD and AD (Hodges et al.,
2004). Indeed, it has been proposed that $\mathrm{AD}$ and FTLD are opposite ends of a spectrum with much overlap clinically, pathologically and genetically (Liscic et al., 2007; van der Zee et al., 2008).

Several scales assess the cognitive profile of frontal executive function and neuropsychiatric symptoms referable to frontal structures. The Frontal Assessment Battery (FAB) (Dubois et al., 2000; Slachevsky et al., 2004) and the EXIT-25 (Royall et al., 1994) assess cognitive functions, particularly dysexecutive features, while the Frontal Behavioral Inventory (FBI) (Kertesz et al., 2000; Marczinski et al., 2004) and the Frontotemporal Behavior Scale (Lebert et al., 1998) assess neuropsychiatric ("behavioral") symptoms. It has previously been demonstrated that $\mathrm{AD}$ patients with greater frontal neuropsychiatric features as measured by higher FBI scores differ from $\mathrm{AD}$ cases with lower FBI scores across a range of features including cognition, function and behavior and more closely resemble subjects with FTLD in several of these features (Woodward et al., 2010b). It was proposed in that study that these "high FBI $A D$ " patients had frontal variant $A D$ (FvAD), an $\mathrm{AD}$ syndrome variant based on clinical features.

We hypothesized that this proposed frontal variant of $\mathrm{AD}$ could also be clinically identified by lower scores on the FAB (indicating poorer executive function) and that these subjects would resemble FTLD subjects and both would differ from other $\mathrm{AD}$ subjects on a range of features including the degree of cognitive, behavioral and functional impairment, use of psychotropic medications, the degree of caregiver burden and the amount of change over 12 months.

\section{Methods}

PRIME (Prospective Research In MEmory clinics, NCT00297271) is an ongoing non-prescriptive, longitudinal convenience cohort study. The primary purpose of PRIME is to provide a cohort of patients that can be examined to quantify complex relationships between a number of interrelated predictor and outcome variables. The study population is representative of Australian dementia patients treated by specialists, working in memory clinics and experienced in dementia management. A description of Australian memory clinics has been recently published (Woodward and Woodward, 2009).

Patients were eligible for inclusion if they had been diagnosed with dementia, by a clinician at a specialized centre of excellence, using the Diagnostic and Statistical Manual of Mental Disorders, 4th edition (DSM-IV; American Psychiatric Association, 2000) criteria or mild cognitive impairment under the Petersen criteria 


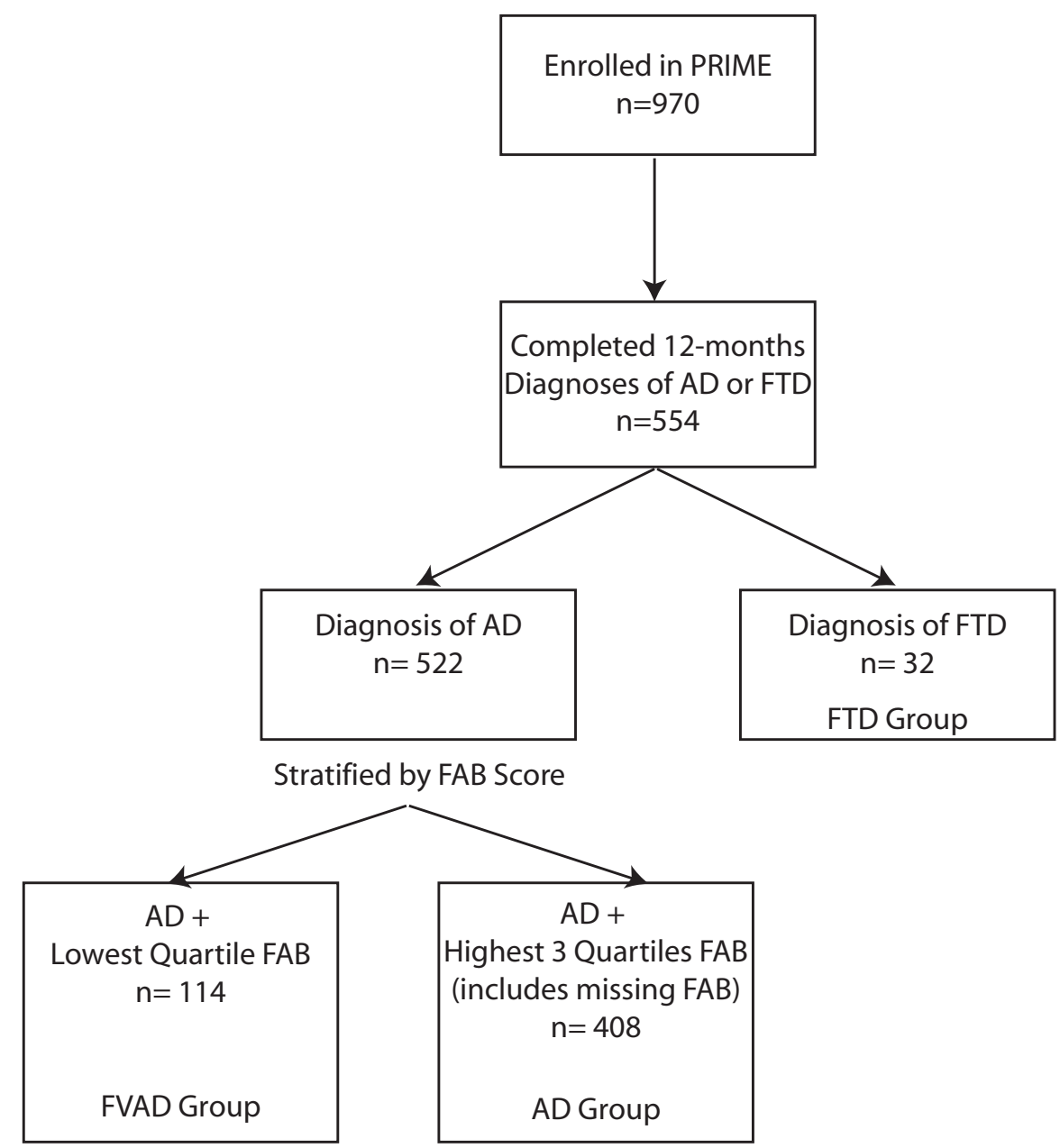

Figure 1. Flowchart of patient selection, whole group, unmatched for severity.

$\mathrm{PRIME}=$ Prospective Research In MEmory clinics; $A D=$ Alzheimer's disease; FTD = frontotemporal dementia; FAB = Frontal Assessment Battery; FVAD = frontal variant Alzheimer's disease.

(Petersen et al., 1999); were living in the community with fewer than 40 hours/week nursing care; had a caregiver willing to provide consent for required components of the study; were fluent in English; and could provide informed consent, or provision of written informed consent by a legal guardian/proxy was obtained. Clinicians utilized all available information to establish a diagnosis, including neuroimaging and neuropsychological assessments where available, and only initial diagnoses were utilized in this study. Patients were excluded if they had any concomitant life-threatening illness which was considered likely to interfere with the patient's ability to complete the study or if they were concurrently participating in a clinical trial of an investigational drug (phase I, II or III).

The following data were prospectively collected: baseline demographics and baseline, 6- and 12-monthly ratings on the Clinical Dementia Rating (CDR; Morris, 1993); Mini-mental State Examination (MMSE; Folstein et al., 1975); Alzheimer's Disease Assessment Scale - Cognitive (ADAS-Cog; Rosen et al., 1984); Functional
Autonomy Measurement System (SMAF; Hebert et al., 1988); Neuropsychiatric Inventory (NPI; Cummings et al., 1994); Clock Drawing Test (Sunderland et al., 1989); Frontal Assessment Battery (FAB), and Zarit Caregiver Burden Interview (ZBI) (Bédard et al., 2001). Instruments were recommended, but not required, to be completed at all visits. Only patients who had been enrolled for 12 months or more were included in this analysis.

For this analysis, patients were grouped by diagnosis. The first group of patients consisted of those with a diagnosis of FTLD as assessed by the clinician, using the Lund-Manchester criteria (Lund and Manchester Groups, 1994). Patients with a diagnosis of $\mathrm{AD}$ were stratified by Frontal Assessment Battery (FAB) scores into two groups, with the quartile with the lowest FAB scores (i.e. reflecting greater executive dysfunction) nominated as the frontal variant Alzheimer's disease (FvAD) group, with the remainder called the $\mathrm{AD}$ group (Figure 1). Patients without a valid recorded baseline $\mathrm{FAB}$ score were included in the $\mathrm{AD}$ group. The data were also analyzed excluding those $\mathrm{AD}$ 
patients with missing FAB scores to determine the sensitivity of the findings to these $\mathrm{AD}$ cases with missing FAB scores.

To assess whether apparent differences between groups were reflecting differences in baseline severity, an additional analysis was conducted with the SMAF used as a marker of severity. Each FTLD subject was matched with four $\mathrm{AD}$ cases with the closest SMAF score, and these four cases were then removed from the pool of $\mathrm{AD}$ cases before the next FTLD case was matched. The AD cases were then arrayed on the basis of their FAB score, with the lowest quartile again nominated as the FvAD group. $\mathrm{AD}$ cases with missing $\mathrm{FAB}$ scores were excluded in this analysis.

\section{Statistical analyses}

All analyses were made pairwise between FTLD and FvAD, FvAD and $A D$ and FTLD and
AD groups respectively. Baseline demographics and assessments, six-month assessments and 12month assessments, and the number of patients institutionalized at 12 months were all examined with Wilcoxon two sample tests or t-tests for continuous data and Fisher's exact test or $\chi^{2}$ for categorical data. Wilcoxon two sample tests were performed upon the individual items of FAB at baseline for the FTLD and FvAD groups. Continuous variables are presented as mean ( \pm standard deviation) and categorical variables are presented as $(\mathrm{n}(\%))$ unless otherwise stated. No a priori adjustments were made for multiplicity and significance was defined at the level of 0.05.

\section{Results}

At the time of this analysis, 523 patients with a diagnosis of $\mathrm{AD}$ and 31 patients with FTLD had

Table 1. Baseline demographics and assessments

\begin{tabular}{|c|c|c|c|c|c|c|}
\hline & FTLD & $\begin{array}{l}\mathrm{p} \text { (FTLD } \\
\text { vFVAD) }\end{array}$ & FVAD & $\begin{array}{l}\mathrm{p}(\mathrm{FVAD} \\
\mathrm{VAD})\end{array}$ & $\mathrm{AD}$ & $\begin{array}{l}p(A D \\
\text { vFTLD) }\end{array}$ \\
\hline $\mathrm{N}$ & 30 & & 114 & & 409 & \\
\hline Age at baseline & $70.9(8.0)$ & $<0.001$ & $78.1(7.4)$ & 0.49 & $78.4(7.7)$ & $<0.001$ \\
\hline Age at onset & $65.1(9.1)$ & $<0.001$ & $73.1(7.9)$ & 0.097 & $74.2(8.4)$ & $<0.001$ \\
\hline Male & $20(66.7 \%)$ & & $48(42.1 \%)$ & 0.14 & $206(50.4 \%)$ & 0.092 \\
\hline Caucasian & $30(100 \%)$ & 0.67 & $109(95.6 \%)$ & 0.45 & $398(97.3 \%)$ & 1.000 \\
\hline \multicolumn{7}{|l|}{ Marital status } \\
\hline Married & $26(86.7 \%)$ & & $84(73.7 \%)$ & & $306(74.8 \%)$ & 0.089 \\
\hline Widowed & $1(3.3 \%)$ & 0.023 & $25(21.9 \%)$ & 0.95 & $75(18.4 \%)$ & \\
\hline Other & $3(10.0 \%)$ & & $5(4.4 \%)$ & & $28(6.9 \%)$ & \\
\hline Family history of dementia & $13(43.3 \%)$ & 0.68 & $44(38.6 \%)$ & 0.74 & $151(36.9 \%)$ & 0.558 \\
\hline \multicolumn{7}{|l|}{ Living arrangement } \\
\hline Alone & $4(13.3 \%)$ & & $14(12.3 \%)$ & & $51(12.5 \%)$ & 0.090 \\
\hline With spouse & $26(86.7 \%)$ & 0.057 & $83(72.8 \%)$ & 0.76 & $307(75.1 \%)$ & \\
\hline Other & $0(0 \%)$ & & $17(14.9 \%)$ & & $51(12.5 \%)$ & \\
\hline Psychotropic medication & $21(70.0 \%)$ & $<0.001$ & $38(33.3 \%)$ & 0.82 & $143(35.0 \%)$ & $<0.001$ \\
\hline Caregiver female & $22(73.3 \%)$ & 0.21 & $68(59.6 \%)$ & 0.51 & $258(63.1 \%)$ & 0.327 \\
\hline \multicolumn{7}{|c|}{ Caregiver relationship to patient } \\
\hline Spouse & $26(86.7 \%)$ & & $80(70.2 \%)$ & & $294(71.9 \%)$ & 0.052 \\
\hline Child & $1(3.3 \%)$ & 0.028 & $28(24.6 \%)$ & 0.59 & $81(19.8 \%)$ & \\
\hline Other & $3(9.9 \%)$ & & $6(5.3 \%)$ & & $34(8.3 \%)$ & \\
\hline Caregiver employed & $4(13.3 \%)$ & 0.60 & $23(20.2 \%)$ & 0.32 & $102(24.9 \%)$ & 0.188 \\
\hline \multicolumn{7}{|l|}{ Baseline assessments } \\
\hline FAB & $14.3(4.5)$ & $<0.001$ & $7.0(2.3)$ & $<0.001$ & $14.6(2.3)$ & 0.362 \\
\hline MMSE & $23.4(4.9)$ & $<0.001$ & $16.6(5.0)$ & $<0.001$ & $22.4(4.7)$ & 0.140 \\
\hline NPI & $25.4(23.8)$ & 0.06 & $16.2(16.8)$ & 0.011 & $12.2(13.4)$ & 0.001 \\
\hline SMAF & $-15.3(10.5)$ & 0.001 & $-23.5(11.3)$ & $<0.001$ & $-16.3(10.4)$ & 0.682 \\
\hline ADAS-Cog & $17.0(6.7)$ & 0.042 & $23.5(7.6)$ & $<0.001$ & $15.2(6.9)$ & 0.423 \\
\hline CDR & $0.9(0.6)$ & 0.002 & $1.3(0.7)$ & $<0.001$ & $0.9(0.5)$ & 0.595 \\
\hline Clock Drawing Test & $8.3(2.6)$ & $<0.001$ & $5.3(2.9)$ & $<0.001$ & $7.7(2.6)$ & 0.126 \\
\hline ZBI & $33.6(19.2)$ & 0.049 & $25.8(13.7)$ & 0.007 & $22.2(15.2)$ & 0.001 \\
\hline
\end{tabular}

FTLD = Frontotemporal Lobar Degeneration; FvAD = Frontal Variant Alzheimer's disease; AD = Alzheimer's disease; FAB = Frontal Assessment Battery; MMSE = Mini-mental state Examination; NPI = Neuropsychiatric Inventory; SMAF = Functional Autonomy Measurement System; ADAS-Cog = Alzheimer's disease Assessment Scale, Cognitive subscale; CDR = Clinical Dementia Rating scale; $\mathrm{ZBI}=$ Zarit Burden Inventory. 
Table 2. Change in assessment scores over 6 and 12 months from baseline

\begin{tabular}{|c|c|c|c|c|c|c|}
\hline & FTLD & $\mathrm{p}($ FTLD v FVAD $)$ & FVAD & $\mathrm{p}(\mathrm{FVAD} \mathrm{V}$ AD) & $\mathrm{AD}$ & $\mathrm{p}(\mathrm{FTLD} \vee \mathrm{AD})$ \\
\hline $\mathrm{N}$ & 30 & & 114 & & 409 & \\
\hline \multicolumn{7}{|c|}{ 6-month change in assessments* } \\
\hline FAB & $-2.3(3.7)$ & 0.001 & $1.0(3.5)$ & 0.002 & $-0.5(3.2)$ & 0.031 \\
\hline MMSE & $-2.6(4.5)$ & 0.498 & $-1.6(3.8)$ & 0.037 & $-0.7(3.2)$ & 0.054 \\
\hline NPI & $6.3(22.8)$ & 0.89 & $2.7(16.8)$ & 0.49 & $2.1(15.0)$ & 0.609 \\
\hline SMAF & $-4.9(8.9)$ & 0.87 & $-4.5(7.4)$ & 0.27 & $-3.2(6.4)$ & 0.873 \\
\hline ADAS-Cog & $-0.3(7.3)$ & 0.76 & $-2.9(2.3)$ & 0.005 & $1.1(5.2)$ & 0.352 \\
\hline CDR & $0.2(0.5)$ & 0.33 & $0.4(0.8)$ & 0.007 & $0.1(0.5)$ & 0.600 \\
\hline Clock Drawing Test & $-0.4(1.6)$ & 0.71 & $-0.6(2.7)$ & 0.199 & $-0.2(2.5)$ & 0.700 \\
\hline ZBI & $2.7(18.0)$ & 0.44 & $5.3(13.7)$ & 0.11 & $2.5(10.8)$ & 0.845 \\
\hline \multicolumn{7}{|c|}{ 12-month change in assessments* } \\
\hline FAB & $-2.4(3.3)$ & 0.003 & $0.2(3.4)$ & 0.07 & $-0.9(3.1)$ & 0.015 \\
\hline MMSE & $-1.9(4.0)$ & 0.18 & $-3.1(4.6)$ & $<0.001$ & $-1.2(3.4)$ & 0.585 \\
\hline NPI & $5.8(25.8)$ & 0.11 & $8.2(23.3)$ & 0.62 & $4.5(16.5)$ & 0.091 \\
\hline SMAF & $-4.8(9.0)$ & 0.018 & $-10.0(10.1)$ & 0.002 & $-5.8(7.6)$ & 0.274 \\
\hline ADAS-Cog & $1.4(5.3)$ & 0.29 & $-1.6(4.3)$ & 0.021 & $3.1(6.2)$ & 0.493 \\
\hline $\mathrm{CDR}$ & $0.3(0.6)$ & 0.103 & $0.5(0.8)$ & 0.001 & $0.2(0.6)$ & 0.809 \\
\hline Clock Drawing Test & $-0.3(1.9)$ & 0.19 & $-1.2(3.3)$ & 0.024 & $-0.4(2.5)$ & 0.658 \\
\hline ZBI & $4.3(22.9)$ & 0.33 & $6.4(18.1)$ & 0.38 & $4.8(14.0)$ & 0.449 \\
\hline
\end{tabular}

${ }^{*}$ Means and standard deviations calculated from available data, missing data ignored.

FTLD = Frontotemporal Lobar Degeneration; FvAD = Frontal variant Alzheimer's disease; $\mathrm{AD}=$ Alzheimer's disease; FAB = Frontal Assessment Battery; MMSE = Mini-mental state Examination; NPI = Neuropsychiatric Inventory; SMAF = Functional Autonomy Measurement System; ADAS-Cog = Alzheimer's disease Assessment Scale, Cognitive subscale; CDR = Clinical Dementia Rating scale; $\mathrm{ZBI}=$ Zarit Burden Inventory.

been enrolled in this study for at least 12 months. One subject with FTLD had no baseline SMAF and as the subject was excluded from the matchedseverity analysis the subject was also excluded from the initial analyses. The baseline demographics and assessments for the three groups, unmatched for severity, are presented in Table 1 along with the results of the pairwise comparisons between groups. In stratifying $\mathrm{AD}$ patients by baseline $\mathrm{FAB}$ scores, the 25 th percentile fell between scores of 10 and 11 . All patients with scores of 10 or less were included in the FvAD group.

The FvAD group had a similar age at both onset and baseline to the $\mathrm{AD}$ group but significantly differed from this group across all assessment scales, showing greater cognitive, functional, neuropsychiatric and global impairment and greater caregiver burden. The FTLD group more closely resembled the $\mathrm{AD}$ group than the FvAD group across all scales, differing significantly from the $\mathrm{AD}$ group in only the NPI and caregiver burden, where the mean scores of the FTLD group were in the direction of the FvAD group and away from that of the $\mathrm{AD}$ group.

Predictably, the FTLD patients were younger than both $\mathrm{AD}$ groups, and had a younger age at symptom onset. They did not resemble the FvAD group across most of the assessment scales, showing fewer frontal features (FAB), less cognitive and global impairment (MMSE, ADAS-Cog, Clock Drawing, CDR) and less functional impairment (SMAF). They did not significantly differ from the FvAD group in neuropsychiatric symptoms (NPI) but subjects with FTLD were associated with greater caregiver burden (Zarit) than subjects with FvAD and were more likely to be receiving psychotropic medication.

The changes in assessment scores and pairwise significance tests at six and 12 months are shown in Table 2 for each patient group. At six and 12 months, subjects with FTLD developed a significantly greater increase in frontal dysexecutive features than both the FvAD and AD groups, whose FAB scores remained relatively stable. There were also differences between the FTLD and FvAD groups at 12 months in the changes in the SMAF, where there was deterioration in both groups, but more so in the FvAD group. In all other scales the FTLD and FVAD groups showed similar changes at both six and 12 months. The FvAD group were found to deteriorate significantly more at six and 12 months than the $\mathrm{AD}$ group on most scales.

Apart from the FTLD group having a significantly greater decline in executive function at six and 12 months than the $\mathrm{AD}$ group, there were insignificant differences between these two groups at 12 months in the changes in the other scales. 
Table 3. Baseline demographics and assessments, matched for severity

\begin{tabular}{|c|c|c|c|c|c|c|}
\hline & FTLD & $\begin{array}{l}\text { p-VALUE } \\
\text { (FTLD } \\
\text { v Fvad) }\end{array}$ & FVAD & $\begin{array}{l}\text { p-VALUE } \\
\text { (FVAD } \\
\text { VAD) }\end{array}$ & $\mathrm{AD}$ & $\begin{array}{l}\mathrm{p}-\mathrm{VALUE} \\
(\mathrm{AD} \\
\mathrm{V} \text { FTLD) }\end{array}$ \\
\hline $\mathrm{N}$ & 30 & & 34 & & 86 & \\
\hline Male $(\%)$ & $20(66.7)$ & 0.115 & $16(47.1)$ & 0.861 & $42(48.8)$ & 0.092 \\
\hline Mean age $(\mathrm{SD})$ & $70.9(8.0)$ & $<0.001$ & $77.8(5.5)$ & 0.624 & $78.4(6.4)$ & $<0.001$ \\
\hline Mean onset age (SD) & $65.1(9.1)$ & $<0.001$ & $73.5(5.7)$ & 0.738 & $74.0(7.5)$ & $<0.001$ \\
\hline Family history of dementia (\%) & $13(43.3)$ & 0.511 & $12(35.3)$ & 0.774 & $28(32.6)$ & 0.288 \\
\hline \multicolumn{7}{|l|}{ Dementia type $(\%)$} \\
\hline $\mathrm{AD}$ (early) & 0 & $<0.001$ & $9(26.5)$ & 0.513 & $18(20.9)$ & $<0.001$ \\
\hline $\mathrm{AD}$ (late) & 0 & & $25(73.5)$ & & $68(79.1)$ & \\
\hline \multicolumn{7}{|l|}{ Living arrangement (\%) } \\
\hline With spouse & $26(86.7)$ & 0.400 & $28(82.4)$ & 0.302 & $67(77.9)$ & 0.055 \\
\hline Alone & $4(13.3)$ & & $4(11.8)$ & & $6(7.0)$ & \\
\hline Other & 0 & & $2(5.9)$ & & $13(15.1)$ & \\
\hline Psychotropic medication use (\%) & $21(70.0)$ & 0.001 & $10(29.4)$ & 0.300 & $34(39.5)$ & 0.004 \\
\hline Caregiver Female & $22(73.3)$ & 0.325 & $21(61.8)$ & 0.823 & $55(64.0)$ & 0.349 \\
\hline Caregiver employed & $4(13.3)$ & 0.564 & $3(8.8)$ & 0.116 & $18(20.9)$ & 0.361 \\
\hline \multicolumn{7}{|l|}{ Baseline Assessment Scores } \\
\hline Mean MMSE (SD) & $23.4(4.9)$ & $<0.001$ & $17.6(5.0)$ & $<0.001$ & $23.2(3.8)$ & 0.818 \\
\hline Mean ADAS-Cog (SD) & $17.0(6.7)$ & 0.194 & $22.3(8.8)$ & $<0.001$ & $13.1(4.5)$ & 0.043 \\
\hline Mean CDR (SD) & $0.9(0.6)$ & 0.591 & $1.0(0.6)$ & 0.011 & $0.7(0.4)$ & 0.087 \\
\hline Mean NPI (SD) & $25.4(23.8)$ & 0.046 & $15.5(13.4)$ & 0.185 & $11.6(14.7)$ & $<0.001$ \\
\hline Mean SMAF (SD) & $-15.3(10.5)$ & 0.077 & $-20.0(10.4)$ & 0.002 & $-13.7(9.7)$ & 0.441 \\
\hline Mean ZBI (SD) & $33.6(19.2)$ & 0.034 & $24.4(14.2)$ & 0.356 & $21.6(15.5)$ & 0.001 \\
\hline Mean FAB (SD) & $14.3(4.5)$ & $<0.001$ & $7.8(2.7)$ & $<0.001$ & $15.3(2.0)$ & 0.116 \\
\hline Mean Clock drawing test (SD) & $8.3(2.6)$ & 0.001 & $5.7(3.0)$ & $<0.001$ & $8.0(2.6)$ & 0.656 \\
\hline
\end{tabular}

FTLD = Frontotemporal Lobar Degeneration; FvAD = Frontal variant Alzheimer's disease; AD = Alzheimer's disease; FAB = Frontal Assessment Battery; MMSE = Mini-mental state Examination; NPI = Neuropsychiatric Inventory; SMAF = Functional Autonomy Measurement System; ADAS-Cog = Alzheimer's disease Assessment Scale, Cognitive subscale; CDR = Clinical Dementia Rating scale; ZBI = Zarit Burden Inventory.

Only a small number of patients were institutionalized and the proportions were similar in both the FTLD and FVAD patient groups at both six months (two patients, $7.4 \%$ versus 11 patients, $12.0 \%, \mathrm{p}=0.73$ ) and 12 months (cumulatively three patients, $11.5 \%$ versus 13 patients, $16.9 \%, \mathrm{p}=0.75$ ). Statistically more FvAD patients were institutionalized at both six (11 patients, $12.0 \%$ versus 19 patients, $5.2 \% \mathrm{p}=$ 0.032 ) and 12 months (13 patients, $16.9 \%$ versus 24 patients, $7.3 \% \mathrm{p}=0.014$ ) compared with the $\mathrm{AD}$ group.

The reanalysis of the data excluding those $\mathrm{AD}$ cases with missing FAB scores showed little change from the results when these were included. Very few $\mathrm{p}$ values for differences between the groups moved over the 0.05 boundary, suggesting the results were not sensitive to the inclusion or exclusion of these patients. The major effect was in the changes over 6 and 12 months (shown in Table 2) where the significant differences between the FvAD and $\mathrm{AD}$ groups were attenuated, with the FvAD group no longer deteriorating significantly more than the $\mathrm{AD}$ group in MMSE and CDR at 6 months and ADAS-Cog and Clock drawing at 12 months.

When we analyzed the data matching for baseline dementia functional severity the FvAD group differed substantially from the AD group across most assessment scales, with the exception of the NPI and the Zarit, and was now much more similar to FTLD on all assessment scales except the MMSE and clock drawing (Tables 3 and 4). As in the analysis not controlled for severity, the FAB score was lower for the FvAD group than for the FTLD group. The FvAD subjects differed from the FTLD group in being older and using fewer psychotropics but were similar to both the other groups on other baseline measures.

Over six and 12 months the matched FvAD group changed to a similar degree in most parameters as the other two groups except that these FvAD cases deteriorated more functionally and globally; they also deteriorated more cognitively than the AD group on the MMSE but not on the ADAS-Cog. The FTLD group was again found to deteriorate more on the FAB than the other two groups. 
Table 4. Change from baseline in assessment scores at 6 and 12 months, matched for severity at baseline

\begin{tabular}{|c|c|c|c|c|c|c|}
\hline & FTLD & $\begin{array}{l}\text { p-VALUE } \\
(\text { FTLD } \\
\text { V FVAD) }\end{array}$ & FVAD & $\begin{array}{l}\mathrm{p}-\text { VALUE } \\
(\text { FVAD } \\
\text { V AD) }\end{array}$ & $\mathrm{AD}$ & $\begin{array}{l}\text { p-VALUE } \\
(\text { FTLD } \\
\text { VAD) }\end{array}$ \\
\hline $\mathrm{n}$ & 30 & & 34 & & 86 & \\
\hline \multicolumn{7}{|c|}{ 6-month change in assessment scores } \\
\hline Mean MMSE (SD) & $-2.6(4.5)$ & 0.172 & $-1.1(3.4)$ & 0.423 & $-0.5(3.2)$ & 0.012 \\
\hline Mean ADAS-Cog (SD) & $-0.3(7.3)$ & 0.936 & $-1.0(0)$ & 0.908 & $-0.6(3.1)$ & 0.877 \\
\hline Mean CDR (SD) & $0.2(0.5)$ & 0.077 & $0.6(0.9)$ & 0.002 & $0.2(0.5)$ & 0.540 \\
\hline Mean NPI (SD) & $6.3(22.8)$ & 0.386 & $1.5(15.9)$ & 0.999 & $1.5(13.0)$ & 0.208 \\
\hline Mean SMAF (SD) & $-4.9(8.9)$ & 0.245 & $-7.7(8.3)$ & 0.001 & $-2.4(6.3)$ & 0.139 \\
\hline Mean ZBI (SD) & $2.7(18.0)$ & 0.936 & $2.3(13.3)$ & 0.643 & $1.0(12.0)$ & 0.606 \\
\hline Mean FAB (SD) & $-2.3(3.7)$ & 0.002 & $1.2(3.7)$ & 0.013 & $-0.5(2.6)$ & 0.009 \\
\hline Mean Clock drawing test (SD) & $-0.4(1.6)$ & 0.807 & $-0.6(3.0)$ & 0.663 & $-0.3(2.4)$ & 0.871 \\
\hline \multicolumn{7}{|c|}{ 12-month change in assessment scores } \\
\hline Mean MMSE (SD) & $-1.9(4.0)$ & 0.106 & $-4.0(4.8)$ & 0.008 & $-1.2(4.1)$ & 0.455 \\
\hline Mean ADAS-Cog (SD) & $1.4(5.3)$ & 0.685 & $-0.3(6.0)$ & 0.473 & $1.5(3.8)$ & 0.970 \\
\hline Mean CDR (SD) & $0.3(0.6)$ & 0.009 & $0.8(0.8)$ & 0.001 & $0.3(0.6)$ & 0.942 \\
\hline Mean NPI (SD) & $5.8(25.8)$ & 0.997 & $5.8(21.9)$ & 0.596 & $3.3(17.9)$ & 0.598 \\
\hline Mean SMAF (SD) & $-4.8(9.0)$ & 0.023 & $-11.9(12.0)$ & 0.002 & $-4.7(8.2)$ & 0.955 \\
\hline Mean ZBI (SD) & $4.3(22.9)$ & 0.395 & $-1.0(18.7)$ & 0.301 & $3.7(17.9)$ & 0.885 \\
\hline Mean FAB (SD) & $-2.4(3.3)$ & 0.044 & $-0.2(3.8)$ & 0.444 & $-0.8(2.8)$ & 0.020 \\
\hline Mean Clock drawing test (SD) & $-0.3(1.9)$ & 0.193 & $-1.4(3.4)$ & 0.227 & $-0.5(2.6)$ & 0.732 \\
\hline
\end{tabular}

FTLD = Frontotemporal Lobar Degeneration; FvAD = Frontal variant Alzheimer's disease; $\mathrm{AD}=$ Alzheimer's disease $; \mathrm{FAB}=\mathrm{Frontal}$ Assessment Battery; MMSE = Mini-mental state Examination; NPI = Neuropsychiatric Inventory; SMAF = Functional Autonomy Measurement System; ADAS-Cog = Alzheimer's disease Assessment Scale, Cognitive subscale; CDR = Clinical Dementia Rating scale; $\mathrm{ZBI}=$ Zarit Burden Inventory.

\section{Discussion}

Consistent with our hypothesis, we confirmed that a subset of $\mathrm{AD}$ patients with greater dysexecutive features differed substantially across a wide range of features from the rest of the subjects with AD. These subjects were more cognitively and functionally impaired, exhibited more behavioral symptoms and showed a greater decline on several measures over six and 12 months. Their greater neuropsychiatric morbidity was associated with greater caregiver burden. These differences were present despite the groups not differing significantly with respect to age, gender, marital status, family history of dementia, living arrangement, caregiver characteristics or use of psychotropic medication. The greater cognitive, functional and behavioral impairment was associated with a greater rate of institutionalization.

These FvAD subjects also differed substantially from the FTLD group in most domains, with the exception of neuropsychiatric symptoms. The FTLD group more closely resembled the $\mathrm{AD}$ group than those with FvAD in all domains except age of onset, neuropsychiatric symptoms, use of psychotropics and caregiver burden. This is a surprising finding of this study as it was hypothesized that the frontal features of the FvAD group would make them similar to the FTLD group. Rather than define a discrete non-frontal $\mathrm{AD}$ group that differs from both the FvAD and FTLD groups, we have separated out an FvAD group that differs substantially from both the other groups. What is perhaps most remarkable is that in using just one measure of impairment, a lower score on the FAB, we have separated off a group of $\mathrm{AD}$ patients who significantly differ from the rest of $\mathrm{AD}$ patients in all other domains that were measured by an impairment scale (cognitive, functional, behavioral/neuropsychiatric and global).

This finding is at variance with the similar Canadian study (Woodward et al., 2010b) that used a different frontal assessment scale - the FBI. In both studies the clinical features at enrolment were measured and utilized in the analyses. In the Canadian study the FTLD and the proposed FvAD groups were similar across several domains including function and neuropsychiatric symptoms but, as in this study they differed with respect to age of onset (younger for the FTLD group) and cognitive impairment (greater in the proposed FvAD group). On nearly all individual FBI items, the FTLD group and the FvAD group were very similar. In essence, the Canadian study showed that the most dissimilar of the three groups was the (non-frontal) AD group 
whereas we have demonstrated in this study that the most dissimilar group was the FvAD group.

A possible explanation for the varying findings from these two studies is that the FBI identifies frontal behavioral impairments whereas the FAB mostly identifies the cognitive dysexecutive features. As FTLD is frequently characterized by early behavioral changes, an FvAD subgroup defined by a scale measuring these behavioral changes could be expected to resemble the FTLD group. Our FTLD group was indeed more behaviorally disturbed than the other groups, as reflected in the higher NPI score, greater use of pychotropics and higher caregiver burden. Dysexecutive features are an early feature of AD but in FTLD these features often present later so an FvAD group defined by executive impairment could be expected to differ from the FTLD cases in earlier stages. In support of this, on the FAB our FTLD group declined far more at six and 12 months than the FvAD group and this greater degree of change was statistically significant at both time points. In essence, the FTLD group, which began with an almost identical FAB score to the (non frontal) AD group and indeed the whole $\mathrm{AD}$ group, was "catching up" with the FvAD group in their degree of executive dysfunction over the subsequent 12 months and leaving behind the $\mathrm{AD}$ group which was deteriorating far less in their FAB score. Thus, the concept that an FvAD group exists may not be disproven by our finding that this group did not initially resemble the FTLD group when FvAD is defined solely by executive dysfunction.

It does seem likely however that the FvAD groups defined by these two different approaches may not be similar groups. Further supporting this is the finding that the mean NPI scores of our FvAD group were lower than those of the FTLD group, suggesting less initial behavioral disturbance. In the Canadian study, the mean NPI scores of both the proposed FvAD group and the FTLD group were almost identical (29.8 and 29.0 respectively), and differed substantially from that of the other subjects with AD (8.3). Indeed, the mean NPI scores of the FTLD and FvAD groups in that study were higher than those of the FTLD subjects in our study (25.4) suggesting considerable behavioral disturbance in both groups in that study. Others have found that, for the same degree of dementia severity, subjects with FTLD have a greater degree of behavioral disturbance than those with $\mathrm{AD}$ (Mendez et al., 1998).

Is there any clinical value in separating off this proposed FvAD group using a measure of executive dysfunction, as we have done? These patients would appear to differ enough, at least initially, from the FTLD subjects not to pose difficulties in the differential diagnosis between $\mathrm{AD}$ and FTLD. They were more impaired across most measures, older and less likely to be on psychotropic medications. Indeed, they seem far easier to differentiate from subjects with FTLD than do those AD cases with prominent early behavioral features (Woodward et al., 2010b). Our findings suggest that where the differential diagnosis of AD and FTLD is being considered, prominent executive dysfunction in the absence of prominent behavioral impairments supports a diagnosis of $\mathrm{AD}$, not FTLD. In essence, the early features of some cases of $\mathrm{AD}$ overlap with features of subjects with FTLD more in the behavioral domain than in the dysexecutive domain.

Our operationalization of FvAD may simply have led to the selection of subjects with more advanced $\mathrm{AD}$. As $\mathrm{AD}$ progresses the characteristic neuropathology becomes more widespread, and can involve the frontal lobes (Braak and Braak 1991). This could be expected to lead to more apparent frontal symptomatology. Our FvAD group had more impairment than the (remaining) AD group on all assessment scales but were not older at either entry into the study or age at onset of symptoms.

Our further analyses, where the AD and FTLD cases were matched for baseline severity, as measured by the SMAF, suggest that baseline severity may have affected the other characteristics of the two AD groups. Both groups are now marginally less affected on almost all parameters, suggesting the matching process eliminated mainly more severely affected cases which had been included before the matching. The biggest effect of this matching process however is that it created an FvAD group that look more like the FTLD than the $\mathrm{AD}$ group, differing less from the FTLD in most baseline assessment scale scores - insignificantly on many parameters when, post hoc, the p-values were adjusted for multiple comparisons. The FTLD and FvAD groups do differ in the degree of executive dysfunction and in two of the cognitive scales, the MMSE and the Clock-drawing score, the latter also reflecting executive function, but they did not differ on the ADAS-Cog. The FvAD group are still significantly more functionally impaired than the $\mathrm{AD}$ group. Their greater cognitive and functional impairment than both the other groups is interesting and suggests that cognitive, functional and executive impairment in the AD group may be linked. Thus, selecting a group that is more impaired on any of the scales will likely lead to the selection of a more severely impaired group of AD patients even when most severely impaired AD cases are excluded from the selection pool. The 6- and 12-month change data still show the FvAD group deteriorating more functionally and globally than the $\mathrm{AD}$ group, and the FTLD group deteriorating more on the FAB than both other groups- again supporting a "catch 
up" in the degree of executive dysfunction in a group that initially and surprisingly showed only as much executive dysfunction as the $\mathrm{AD}$ group.

On balance, our findings seem to indicate a greater disease burden in the FvAD group but they are also consistent with the FvAD group having a distinct clinical presentation characterized by prominent executive dysfunction but no difference in disease duration or age of onset to the other $\mathrm{AD}$ cases. The further severity-matched analysis has not changed this essential difference between the FvAD and the $\mathrm{AD}$ cases, or the similarity between the $\mathrm{AD}$ and FTLD groups, but has created an FvAD group that now looks more like FTLD and, as in the other study (Woodward et al., 2010b) still raises the possibility that there may be a frontal variant of $\mathrm{AD}$ that looks more like FTLD than AD.

We concede that there are limitations to our findings. There could have been selection bias in the administration of the FAB within the study as the protocol did not require all subjects to have all scales administered at all visits. We did identify 139 subjects who did not have the FAB administered at baseline. There is also clearly circularity in selecting FvAD cases according to a frontal dysexecutive score and then characterizing FvAD thus defined on this and other clinical measures. There is, however, justification for examining subjects at the statistical margins of any disease, rather than just the "average" patient. The circularity, however, and a limited availability of information around the presenting complaints of our FvAD cases and their longitudinal change prior to entry into our study limit the clinical validity of our observations. Additionally, we relied on clinical diagnosis to assign diagnostic categories, which may well have been incorrect in some cases. To somewhat offset this risk, the clinicians were working in centers of excellence with access to neuroimaging and other assessments, which have been shown to produce a diagnostic accuracy, when correlated with autopsy data, of around 90\% (Lopez et al., 2000). Penultimately, even matching the AD cases to the FTLD cases for baseline functional severity and then differentiating a subgroup with the lowest FAB scores made it difficult to differentiate the effects of severity from type as we again separated the most severely dementing AD cases. Lastly, there was no neuropathological confirmation of the clinical diagnoses presented in this study.

Notwithstanding these limitations, our data are consistent with the existence of a subgroup of AD patients with greater executive dysfunction who are probably a more severely affected group of those with $\mathrm{AD}$ and who do not initially have a strong clinical overlap with the subjects with FTLD, who had a greater degree of behavioral disturbance. It is only when baseline severity is matched that this FvAD group resembles the FTLD group, and such matching is impractical in the clinical setting. The study does encourage future prospective clinical studies aimed at the further characterization of FvAD, however defined, and investigations into the neuropathological basis of this proposed variant of AD. Clinicians should be cautious when assigning patients with dementia and behavioral or executive dysfunction to a diagnosis of either FTLD or AD. Clinical differentiation can be difficult and the future may lie in combining clinical assessment with other investigations such as biomarkers.

\section{Conflict of interest declaration}

The first four authors have all been members of advisory boards funded by the companies marketing galantamine, donepezil and rivastigmine. They have been members of the PRIME database Scientific Advisory Committee (SAC), funded by JanssenCilag Pty Ltd Australia, and were funded for this activity. Greg Blanch is an employee of JanssenCilag Pty Ltd Australia and Robert Balshaw is an employee of Syreon Corporation, Canada, which processed the data and was paid by Janssen-Cilag Pty Ltd Australia to do this.

Because Professor Ames is also Editor-in-Chief of International Psychogeriatrics, the peer review process was handled entirely by one of the Journal's Deputy Editors.

\section{Description of authors' roles}

Michael Woodward designed the concept, directed the statistical analyses and wrote the majority of the paper. Henry Brodaty, Karyn Boundy and David Ames contributed to the interpretation of the data and the final version of the paper. Greg Blanch contributed to the final draft of the paper but did not direct content. Robert Balshaw and Syreon Corporation assisted with statistical analyses, as directed by the Scientific Advisory Committee.

\section{Acknowledgments}

We thank Andrea Malcolm (Janssen-Cilag Australia Pty Ltd) for assistance during the preparation of this paper and Craig Batty for project management assistance with the PRIME data base (through Janssen-Cilag Australia Pty Ltd).

\section{References}

American Psychiatric Association (2000). Diagnostic and Statistical Manual of Mental Disorders, 4th edn, text revision 
(DSM-IVTR). Washington, DC: American Psychiatric Association.

Becker, J. T., Huff, F. J., Nebes, R. D., Holland, A. and Boller, F. (1998). Neuropsychological function in Alzheimer's disease: pattern of impairment and rates of progression. Archives of Neurology, 45, 263-268.

Bédard, M., Molloy, D. W., Squire, L., Dubois, S., Lever, J. A. and O'Donnell, M. (2001). The Zarit Burden Interview: a new short version and screening version. The Gerontologist, 41, 652-657.

Binetti, G., Magri, E., Padovani, A., Cappa, S. F., Bianchetti, A. and Trabucchi, M. (1996). Executive dysfunction in early Alzheimer's disease. Fournal of Neurology, Neurosurgery and Psychiatry, 60, 91-93.

Braak, H. and Braak, E. (1991). Neuropathological staging of Alzheimer-related changes. Acta Neuropathologica (Berlin), 82, 239-259.

Cairns, N. J., Taylor-Reinwald, L., Morris, J. C. and the Alzheimer's Disease Neuroimaging Initiative (2010). Autopsy consent, brain collection, and standardized neuropathologic assessment of the ADNI participants: the essential role of the Neuropathology Core. Alzheimer's and Dementia, 6, 274-279.

Cummings, J. L., Mega, M., Gray, K., RosenbergThompson, S., Carusi, D. A. and Gorbein, J. (1994). The Neuropsychiatric Inventory: comprehensive assessment of psychopathology in dementia. Neurology, 44, 2308-2314.

Dubois, B., Slachevsky, A., Litvan, I. and Pillon, B. (2000). The FAB: a frontal assessment battery at bedside. Neurology, 55, 1621-1626.

Folstein, M. F., Folstein, S. E. and McHugh, P. R. (1975). "Mini-mental state": a practical method of grading the cognitive status of patients for the clinician. Fournal of Psychiatry Research, 12, 189-198.

Forman, M. S. et al. (2006). Frontotemporal dementia: clinicopathological correlates. Annals of Neurology, 59, 952-962.

Galton, C. J., Patterson, K., Xuereb, J. H. and Hodges, J. R. (2000). Atypical and typical presentations of Alzheimer's disease: a clinical, neuropsychological, neuroimaging and pathological study of 13 cases. Brain, 123, 484-498.

Gorno-Tempini, M. L. et al. (2008). The logopenic/phonological variant of primary progressive aphasia. Neurology, 71, 1227-1234.

Grady, C. L. et al. (1998). Longitudinal study of the early neuropsychological and cerebral metabolic changes in dementia of the Alzheimer type. Fournal of Clinical and Experimental Neuropsychology, 19, 576-596.

Greene, J. D. W., Patterson, K., Xuereb, J. and Hodges, J. R. (1996). Alzheimer's disease and confluent progressive aphasia. Archives of Neurology, 53, 1072-1078.

Hebert, R., Carrier, R. and Bilodeau, A. (1998). The Functional Autonomy Measurement System (SMAF): description and validation of an instrument for the measurement of handicaps. Age and Ageing, 17, 293-302.

Hodges, J. R. et al. (2004). Clincopathological correlates in frontotemporal dementia. Annals of Neurology, 56, 399-406.

Ikeda, M., Ishikawa, T. and Tanabe, H. (2004). Epidemiology of frontotemporal lobar degeneration.
Dementia and Geriatric Cognitive Disorders, 17, 265268.

Johnson, J. K., Head, E., Kim, R., Stark, A., Arnold, M. D. and Cotman, C. W. (1999). Clinical and pathological evidence for a frontal variant of Alzheimer's disease. Neurology, 56, 1233-1239.

Kanne, S. M., Balota, D. A., Storandt, M., McKeel, D. W. Jr, and Morris, J. C. (1998). Relating anatomy to function in Alzheimer's disease: neuropsychological profiles predict regional neuropathology 5 years later. Neurology, 50, 979-985.

Kertesz, A., Nadkarni, N., Davidson, W. and Thomas, A. W. (2000). The Frontal Behavioural Inventory in the differential diagnosis of frontotemporal dementia. Fournal of the International Neuropsychological Society, 6, 460-468.

Kertesz, A., McMonagle, P., Blair, M., Davidson, W. and Munoz, D. G. (2005). The evolution and pathology of frontotemporal dementia. Brain, 128, 1996-2005.

Knibb, J. A., Xuereb, J. H., Patterson, K. and Hodges, J. R. (2006). Clinical and pathological characterization of progressive aphasia. Annals of Neurology, 59, 156-165.

Knopmann, D. S. et al. (2005). Antemortem diagnosis of frontotemporal lobar degeneration. Annals of Neurology, 57, 480-488.

Lebert, F., Pasquier, F., Souliez, L. and Petit, H. (1998). Frontotemporal behavioural scale. Alzheimer's Disease and Associated Disorders, 12, 335-339.

Liscic, R. M., Storandt, M., Cairns, N. J. and Morris, J. C. (2007). Clinical and psychometric distinction of frontotemporal and Alzheimer dementias. Archives of Neurology, 64, 535-540.

Lopez, O. L. et al. (2000). Research evaluation and diagnosis of probable Alzheimer's disease over the last two decades: I. Neurology, 55, 1854-1862.

Lund and Manchester Groups (1994). The Lund Manchester Consensus Statement: clinical and neuropathological criteria for frontotemporal dementia. fournal of Neurology, Neurosurgery and Psychiatry, 57, 416-418.

Marczinski, C. A., Davidson, W. and Kertesz, A. (2004). A longitudinal study of behaviour in frontotemporal dementia and primary progressive aphasia. Cognitive and Behavioral Neurology, 17, 185-190.

Mega, M. S. et al. (1996). The spectrum of behavioural changes in Alzheimer's disease. Neurology, 46, 130-135.

Mendez, M. F., Perryman, K. M., Miller, B. L. and Cummings, J. L. (1998). Behavioural differences between frontotemporal dementia and Alzheimer's disease: a comparison on the BEHAVE-AD rating scale. International Psychogeriatrics, 10, 155-162.

Morris, J. C. (1993). The Clinical Dementia Rating (CDR): current version and scoring rules. Neurology, 43: 2412-2414.

Petersen, R. C., Smith, G. E., Waring, S. C., Ivnik, R. J., Tangalos, E. G. and Kokmen, E. (1999). Mild cognitive impairment: clinical characterization and outcome. Archives of Neurology, 56, 303-308.

Price, B. H., Gurvit, H., Weintraub, S., Geula, C., Leimkuhler, E. and Mesulam, M. (1993).

Neuropsychological patterns and language deficits in 20 consecutive cases of autopsy-confirmed Alzheimer's disease. Archives of Neurology, 50, 931-937. 
Rosen, W. G., Mohs, R. C. and Davis, K. L. (1984). A new rating scale for Alzheimer's disease. American fournal of Psychiatry, 141, 356-364.

Royall, D. R., Mahurin, R. K. and Cornell, J. (1994). Bedside assessment of frontal degeneration: distinguishing Alzheimer's disease from non-Alzheimer's cortical dementia. Experimental Aging Research, 20, 95-103.

Slachevsky, A., Villalpando, J. M., Sarazin, M., Hahn-Barma, V., Pillon, B. and Dubois, B. (2004). Frontal Assessment Battery and differential diagnosis of fronto-temporal dementia and Alzheimer's disease. Archives of Neurology, 61, 1104-1107.

Stern, Y., Mayeux, R., Sano, M., Hauser, W. A. and Bush, T. (1987). Predictors of disease course in patients with probable Alzheimer's disease. Neurology, 37, 1649-1653.

Storey, E., Slavin, M. and Kinsella, G. J. (2002). Patterns of cognitive impairment in Alzheimer's disease: assessment and differential diagnosis. Frontiers in Bioscience, 7, 155-184.

Sunderland, T. et al. (1989). Clock drawing in Alzheimer's disease: a novel measure of dementia severity. Fournal of the American Geriatrics Society, 37, 725-729. van der Zee, J., Sleegers, K. and Van Broeckhoven, C. (2008). The Alzheimer disease frontotemporal lobar degeneration spectrum. Neurology, 71, 1191-1197.

Varma, A. R., Snowden, J. S., Lloyd, J. J., Talbot, P. R., Mann, D. M. A. and Neary, D. (1999). Evaluation of the NINCDS-ADRDA criteria in the differentiation of Alzheimer's disease and frontotemporal dementia. fournal of Neurology, Neurosurgery and Psychiatry, 66, 184-188.

Welsh, K., Butters, N., Hughes, J., Mohs, R. and Heyman, A. (1991). Detection of abnormal memory decline in mild cases of Alzheimer's disease using CERAD neuropsychological measures. Archives of Neurology, 48, 278-281.

Woodward, M. C. and Woodward, E. (2009). A national survey of memory clinics. International Psychogeriatrics, 21, 696-702

Woodward, M. C., Hsiung, R. G.-K., Feldman, H., Jacova, C. and Mackenzie, I. R. (2010a). High prevalence of multiple brain pathologies in dementia. European Geriatric Medicine (in press).

Woodward, M. C., Jacova, C., Black, S., Kertesz, A., Mackenzie, I. R. and Feldman, H. H. (2010b). Differentiating the frontal variant of Alzheimer's disease. International Fournal of Geriatric Psychiatry, 25, 732-738. 


\section{University Library}

\section{- M M I N E R VA A gateway to Melbourne's research publications}

Minerva Access is the Institutional Repository of The University of Melbourne

Author/s:

Woodward, M;Brodaty, H;Boundy, K;Ames, D;Blanch, G;Balshaw, R

Title:

Does executive impairment define a frontal variant of Alzheimer's disease?

Date:

2010-12-01

Citation:

Woodward, M., Brodaty, H., Boundy, K., Ames, D., Blanch, G. \& Balshaw, R. (2010). Does executive impairment define a frontal variant of Alzheimer's disease?. INTERNATIONAL PSYCHOGERIATRICS, 22 (8), pp.1280-1290. https://doi.org/10.1017/S1041610210001596.

Publication Status:

Published

Persistent Link:

http://hdl.handle.net/11343/32987 